\title{
A randomised controlled trial of hearing and vision support in dementia: Protocol for a process evaluation in the SENSE-Cog trial
}

Iracema Leroi ${ }^{1,2^{*}}$ (D), Christopher J. Armitage ${ }^{3}$, Fidéline Collin ${ }^{4}$, Eric Frison ${ }^{4}$, Mark Hann $^{5}$, Emma Hooper ${ }^{2}$, David Reeves ${ }^{5}$, Zoe Simkin ${ }^{2}$, Lucas Wolski ${ }^{6}$ and on behalf of the SENSE-Cog Work Package 3 Investigators

\begin{abstract}
Background: Optimising hearing and vision function may be important in improving a range of outcomes for people living with dementia (PwD) and their companions. The SENSE-Cog cross-national randomised controlled trial (RCT) is evaluating the effectiveness of a sensory intervention (SI) to improve quality of life for PwD with concurrent hearing and/or vision impairment, in five European countries. To ascertain how or why the intervention will, or will not, achieve its outcomes, we have designed a process evaluation to explore potential discrepancies between expected and observed outcomes. This will also help us to understand how context may influence the outcomes. Here we describe the protocol for this process evaluation, which is embedded within the RCT.

Methods/design: We will use a mixed methods approach with a theoretical framework derived from the UK Medical Research Council's' guidance on process evaluations. It will include the following: (1) evaluating how key aspects of the intervention will be delivered, which will be important to scale the intervention in real world populations; (2) characterising the contextual issues, which may shape the delivery and the impact of the intervention in different countries; and (3) investigating possible causal mechanisms through analyses of potential moderators and mediators. To avoid bias, we will analyse the process data before the analysis of the main effectiveness outcomes.

Discussion: This evaluation will provide insight into how the complex SENSE-Cog SI will be tailored, enacted and received across the different European contexts, all of which have unique health and social care economies. The findings will provide insight into the causal mechanisms effecting change, and will determine whether we should implement the intervention, if effective, on a wider scale for PwD and concurrent sensory impairment.
\end{abstract}

Trial registration: ISRCTN, ISRCTN17056211. Registered on 19 February 2018.

Keywords: Process evaluation, Complex intervention, Dementia, Sensory impairment, Randomised controlled trial, Hearing impairment, Vision impairment, Mediators, Moderators, Logic model

\footnotetext{
* Correspondence: iracema.leroi@tcd.ie

${ }^{1}$ Global Brain Health Institute, School of Medicine, Trinity College Dublin,

Trinity College Institute of Neurosciences, Room 0.60, Lloyd Building, Dublin

2 , Ireland

${ }^{2}$ Division of Neuroscience and Experimental Psychology, University of

Manchester and the Manchester Academic Health Sciences Centre,

Manchester, UK

Full list of author information is available at the end of the article
}

(C) The Author(s). 2020 Open Access This article is distributed under the terms of the Creative Commons Attribution 4.0 International License (http://creativecommons.org/licenses/by/4.0/), which permits unrestricted use, distribution, and reproduction in any medium, provided you give appropriate credit to the original author(s) and the source, provide a link to the Creative Commons license, and indicate if changes were made. The Creative Commons Public Domain Dedication waiver (http://creativecommons.org/publicdomain/zero/1.0/) applies to the data made available in this article, unless otherwise stated. 


\section{Background}

There is growing evidence that people with dementia (PwD) with ageing-related hearing and vision impairment experience worse outcomes compared to PwD with optimal sensory function. These outcomes include increased disorientation, difficulties self-locating using visual or auditory cues, higher levels of distress leading to agitation and aggression and increased prevalence of hallucinations, delusions and depression [1-3]. Sensory impairment itself can worsen cognitive decline, as well as exacerbate the social isolation that is often associated with dementia [4]. Individuals may withdraw from social activities and hobbies and become marginalised [5-8]. Furthermore, burnout and physical exhaustion in care partners can be amplified by communication barriers [3] and greater dependency of the $\mathrm{PwD}$. Thus, we designed the SENSE-Cog randomised controlled trial (RCT) to answer the research question, 'Can a home-based, tailored 'sensory intervention' (SI) improve quality of life in PwD with comorbid hearing and/or visual impairment?'

The multi-component SI that the SENSE-Cog trial will evaluate comprises assessment, treatment and support of hearing and vision impairment in PwD. We developed the SI iteratively over 24 months. This involved: (1) a scoping review of the literature [9]; (2) an in-depth qualitative exploration of the support care needs of $\mathrm{PwD}$ with sensory impairment in three European countries [10]; (3) an international survey $(n=653)$; and (4) an interdisciplinary Expert Reference Group $(n=17)$ [11, 12]. The findings were synthesized into a draft SI that was then field tested in the UK, France and Cyprus [1315], prior to development of the protocol for the full multi-site RCT [16], the SENSE-Cog trial.

The SENSE-Cog trial is a 36-week parallel-group, observer-blind, multicentre, superiority RCT comparing the individualised SI to usual care in PwD with hearing and/or visual impairment and their companion (the participant 'dyad'). Briefly, it involves 354 randomized dyads $(1: 1 ; 177$ per arm) in five European sites: Athens (Greece), Dublin (Ireland), Manchester (UK), Nice (France), and Nicosia (Cyprus). The primary outcome of the trial is quality of life in the $\mathrm{PwD}$, measured at 36 weeks post-baseline using the DEMQOL [17]. The DEMQOL is a 29-item, interviewer-administered, selfreport questionnaire with good psychometric properties in persons with mild to moderate dementia. Secondary outcomes include neuropsychiatric symptoms, measures of mental wellbeing, sensory and cognitive functional ability, relationships and health resource utilisation. Companion outcomes and health economic measures are also being assessed. Here we describe the protocol for a process evaluation of the RCT as per the UK Medical Research Council (MRC) recommendations [18].
'Complex interventions', such as the SENSE-Cog SI, are defined as those comprising multiple components interacting to produce change $[18,19]$. The range of outcomes, and the degree of tailoring or flexibility required for each individual participant, is significant, thus adding to the complexity [12]. While RCTs are the soundest means of inferring causality of an intervention, they cannot ascertain how or why an intervention may or may not achieve the outcomes [20]. Thus, we have embedded a detailed process evaluation within the RCT to clarify this [18]. This evaluation, together with the outcomes of the RCT, will enable policy makers, funders and practitioners to determine whether the intervention is effective or not, and whether it should be implemented on a wider scale. This is particularly relevant for the cross-national SENSE-Cog trial, which is taking place in five European contexts. It will fill a significant evidence gap in the management of hearing and vision impairment in PwD.

The specific aims of this process evaluation are to: (1) explore the delivery, or the process through which the SI will be offered, including barriers and facilitators; (2) evaluate contextual issues and clarify factors that may affect the SI delivery, mechanisms and outcomes [21]; and (3) investigate possible causal mechanisms, using analyses of potential moderators and mediators.

\section{Methods/design}

The protocol for our process evaluation follows a systematic approach for the design and conduct of the evaluation [18].

\section{Planning the process evaluation Working with intervention developers and implementers} A process evaluation requires a degree of independence to appraise the intervention team's delivery of the trial [18]. Thus, we delegated oversight of the evaluation to an expert not involved in the day-to-day conduct of the RCT but on the wider SENSE-Cog team (CA). Regular process evaluation reports will be made at Trial Steering Committee meetings [16] and specific meetings with the study chief investigator (IL), the methodologist (EF) and the process lead (CA).

\section{Overlap of the process and outcomes and cost-effectiveness evaluations}

Due to the complexity of the study, we have embedded the process evaluation within the daily conduct of the outcomes and cost-effectiveness evaluations. Thus, data for all three purposes are being collected concurrently, and some measures may be used for both process and outcome evaluations. During team training, we emphasized the multi-purposing of data and the need to maintain 'researcher equipoise'. 


\section{Description of the intervention}

We have previously detailed the SI components and implementation [12]. In summary, the SI, which is delivered as a ten-session programme by a trained 'sensory support therapist' (SST) and audiologists/optometrists, is outlined in Fig. 1. It involves several components, notably: (1) identifying and correcting any vision or hearing impairment; (2) supporting adherence to the hearing and/or vision devices, through advice and training in correct use and care; (3) enhancing communication between the PwD and their companion; (4) demonstrating environmental aids and sensory devices; and (5) accessing relevant support services and social networks. The non-intervention group receives 'care as usual' (CAU) access to the services and interventions normally available to $\mathrm{PwD}$ and their companions in their respective countries and sites.

\section{Causal assumptions about how change will be produced}

To understand fully the impact of the intervention on the outcomes, and to generalise the findings in the panEuropean context of SENSE-Cog, an exploration of causal mechanisms and unanticipated pathways is needed [19]. We will do this by extracting pre-specified mediating variables and qualitative data (from a subsample of 30 participant dyads) and evaluating adverse events and unexpected consequences of the intervention. We will also undertake semi-structured interviews with the SSTs at each of the five study sites.

The principal aim of the intervention is to improve quality of life and functional ability by improving sensory function through devices and behavioural change. Dementia-related quality of life comprises the domains of daily activities (activities of daily living and self-care), physical health and wellbeing, cognitive functioning and

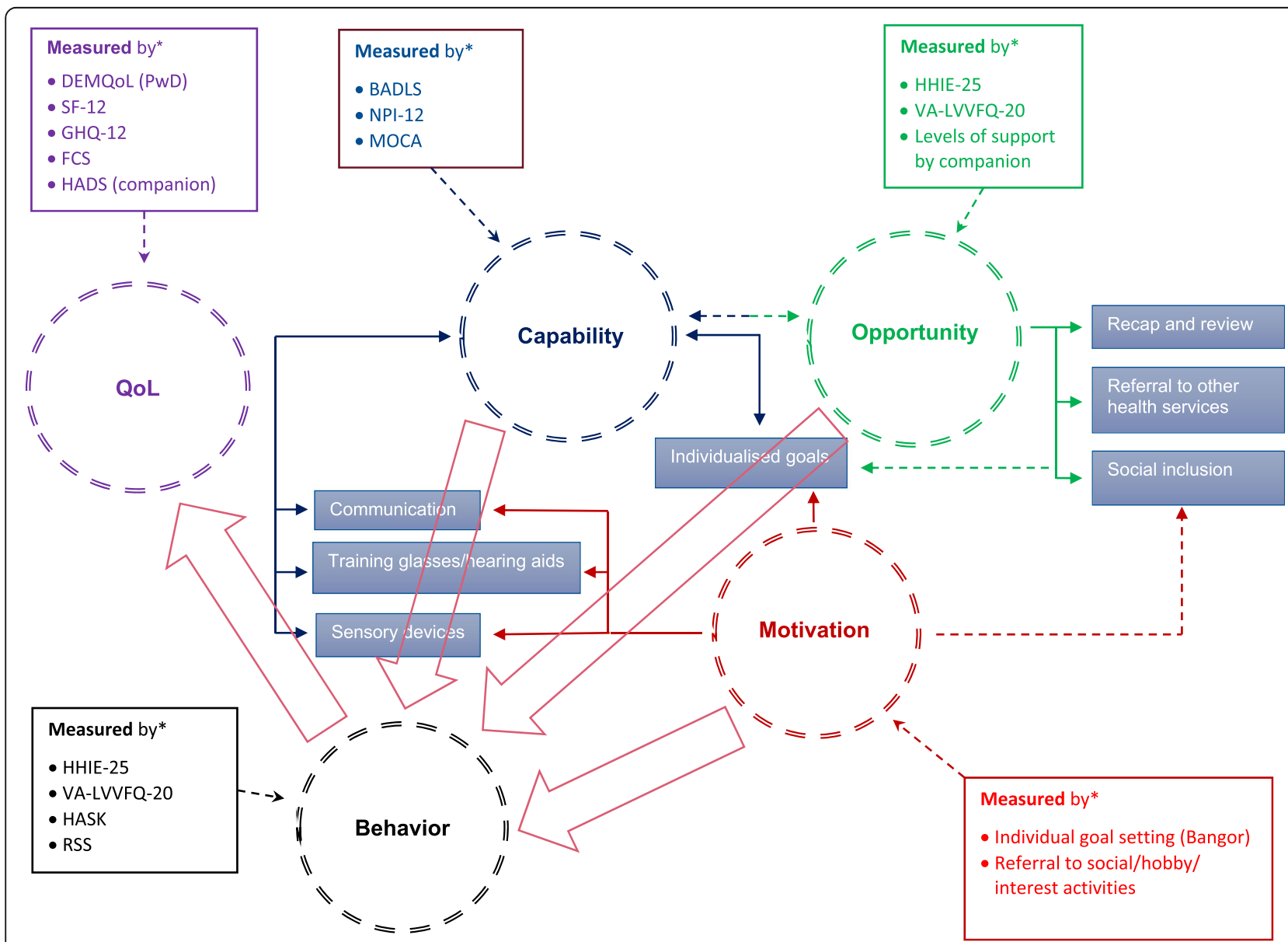

Fig. 1 How the elements of the 'COM-B Behaviour Change' model and components of the sensory intervention link together. BADLs Bristol Activities of Daily Living Scale [22], Bangor The Bangor Goal-Setting interview [23], DEMQoL Dementia Quality of Life [24], FCS Family Caregiving Role Scale [25], HADS Hospital Anxiety and Depression Scale [26], HASK Hearing Aid Skills and Knowledge test [27], HHIE-25 Hearing Handicap Inventory for the Elderly [28], GHQ-12 General Health Questionnaire [29], MoCA Montreal Cognitive Assessment [30], NPI-12 Neuropsychiatric Inventory [31], RSS Relationship Satisfaction Scale [32], SF-12 Short Form Health Survey [33], VA LV-VFQ-20 Veterans Affairs Low vision Visual Functioning Questionnaire [34] 
social relationships [35]. To influence these domains, we have adopted the COM-B component of the Behaviour Change Wheel [36] as our framework for how the intervention might work. According to this model, behavioural change (' $\mathrm{B}$ ') results from: capability ('C'), the individual's psychological and physical capacity to engage in the activity concerned; opportunity ('O'), the external factors that support behavioural change; and motivation (' $\mathrm{M}$ '), the conscious and sub-conscious processes that direct decision making [36]. In Table 1 and Fig. 1, using a logic model, we outline how each COM-B element aligns with specific components of the SI, and how these might hypothetically lead to improvements in quality of life, our primary outcome.

Briefly, for capability, the sensory aids (i.e. hearing aids, glasses and sensory environment modification in the home) and SST adherence support will improve hearing and vision (physical capability), which will enhance sensory-cognitive function and overall functional ability and reduce neuropsychiatric symptoms [37-41]. The SST will train communication skills and improve knowledge of dementia and sensory impairment (psychological capability). This will increase opportunity by decreasing dependency on companions, enhancing social interactions and reducing loneliness. Adherence support for PwD with sensory devices will enhance hearing [42], vision [43] or both [15]. Furthermore, social opportunities will be enhanced through signposting outside the home, thus addressing social isolation, improving social relationships and providing respite for companions. Regarding motivation, higher sensory-cognitive function and improved neuropsychiatric symptoms will improve self-efficacy, self-esteem and mental wellbeing [44, 45]. However, motivation may be reduced in dementia, particularly if apathy is present. Thus, the SI will address this through goal setting. We will measure apathy to

Table 1 Theoretical basis and logic model for how the sensory intervention may impact the person with dementia

\begin{tabular}{|c|c|c|c|c|c|}
\hline Theoretical COM-B domain & $\begin{array}{l}\text { Component of the sensory } \\
\text { intervention }\end{array}$ & $\begin{array}{l}\text { Behaviour change (B) } \\
\text { due to the intervention }\end{array}$ & $\begin{array}{l}\text { Immediate impact of } \\
\text { the behaviour change } \\
\text { (secondary outcomes } \\
\text { of interest) }\end{array}$ & $\begin{array}{l}\text { Intermediate impact of } \\
\text { the behaviour change } \\
\text { (secondary outcomes of } \\
\text { interest) }\end{array}$ & $\begin{array}{l}\text { Primary } \\
\text { outcome } \\
\text { of } \\
\text { interest }\end{array}$ \\
\hline \multirow{4}{*}{$\begin{array}{l}\text { Capability (C) } \\
\text { (the individual's } \\
\text { psychological and physical } \\
\text { capacity to engage in the } \\
\text { activity concerned) }\end{array}$} & $\begin{array}{l}\text { Correct visual / auditory } \\
\text { impairment }\end{array}$ & \multirow{4}{*}{$\begin{array}{l}\text { - Hear and see better } \\
\text { (HHIE-25;VA-LWFQ-20) } \\
\text { - Uptake and adherence } \\
\text { improves } \\
\text { (HASK) } \\
\text { - Greater understanding } \\
\text { /insight of impairments } \\
\text { - Dyadic interaction } \\
\text { improves (RSS) } \\
\text { - Enhanced cognitive } \\
\text { stimulation }\end{array}$} & \multirow{9}{*}{$\begin{array}{l}\text { - Enhanced cognitive } \\
\text { functioning (MoCA) } \\
\text { - Reduced } \\
\text { neuropsychiatric } \\
\text { symptoms (NPI-12) } \\
\text { - Less loneliness in } \\
\text { dyad } \\
\text { - Enhanced social } \\
\text { interactions } \\
\text { - Greater } \\
\text { independence for } \\
\text { person with } \\
\text { dementia } \\
\text { - Greater companion } \\
\text { respite }\end{array}$} & \multirow{9}{*}{$\begin{array}{l}\text { - Enhanced self-efficacy } \\
\text { - Improved } \\
\text { communication } \\
\text { - Greater functional } \\
\text { ability (BADLS) } \\
\text { - Reduced apathy (NPI- } \\
\text { 12) } \\
\text { - Relationship satisfaction } \\
\text { is higher (RSS) in both } \\
\text { person with dementia } \\
\text { and companion } \\
\text { - Companions health } \\
\text { and well-being (SF-12; } \\
\text { GHQ-12; FCS; HADS) }\end{array}$} & \multirow{9}{*}{$\begin{array}{l}\text { - Quality } \\
\text { of life in } \\
\text { PwD } \\
\text { (DEMQoL) }\end{array}$} \\
\hline & $\begin{array}{l}\text { Training in correct use of } \\
\text { hearing aid /glasses with } \\
\text { dyad }\end{array}$ & & & & \\
\hline & $\begin{array}{l}\text { Communication Training } \\
\text { with dyad }\end{array}$ & & & & \\
\hline & $\begin{array}{l}\text { Home-based functional } \\
\text { assessment and tailored } \\
\text { interventions to address } \\
\text { support care needs enabled }\end{array}$ & & & & \\
\hline \multirow[t]{3}{*}{$\begin{array}{l}\text { Opportunity (O) } \\
\text { (external factors which } \\
\text { impact on the individual's } \\
\text { ability to 'live well' with } \\
\text { dementia) }\end{array}$} & $\begin{array}{l}\text { Provision of supplementary } \\
\text { sensory devices }\end{array}$ & $\begin{array}{l}\text { - Hear and see better } \\
\text { (HHIE-25;VA-LWFQ-20) } \\
\text { - Appropriate levels of } \\
\text { support from } \\
\text { companion }\end{array}$ & & & \\
\hline & $\begin{array}{l}\text { Referral to health and social } \\
\text { care services }\end{array}$ & $\begin{array}{l}\text { - Uptake of health and } \\
\text { social care services }\end{array}$ & & & \\
\hline & $\begin{array}{l}\text { Referral to social/hobby/ } \\
\text { interest activities }\end{array}$ & $\begin{array}{l}\text { - Uptake of social/ } \\
\text { hobby/ interest } \\
\text { opportunities }\end{array}$ & & & \\
\hline \multirow[t]{2}{*}{$\begin{array}{l}\text { Motivation (M) } \\
\text { (in dementia, apathy and } \\
\text { loss of motivation is } \\
\text { prevalent and may make } \\
\text { behavioural change difficult) }\end{array}$} & Individualised goal setting & $\begin{array}{l}\text { - Enhanced cognitive } \\
\text { stimulation } \\
\text { - Achievement of set } \\
\text { goals }\end{array}$ & & & \\
\hline & $\begin{array}{l}\text { Referral to social/hobby/ } \\
\text { interest activities }\end{array}$ & $\begin{array}{l}\text { - Enhanced uptake and } \\
\text { adherence of sensory } \\
\text { aids and suggested } \\
\text { social opportunities } \\
\text { - Uptake of meaningful } \\
\text { and enjoyable social/ } \\
\text { hobby/interest } \\
\text { activities }\end{array}$ & & & \\
\hline
\end{tabular}

BADLs Bristol Activities of Daily Living Scale [22], DEMQoL Dementia Quality of Life [24], FCS Family Caregiving Role Scale [25], HADS Hospital Anxiety and Depression Scale [26], HASK Hearing Aid Skills and Knowledge Test [27], HHIE-25 Hearing Handicap Inventory for the Elderly [28], GHQ-12 General Health Questionnaire [29], MoCA Montreal Cognitive Assessment [30], NPI-12 Neuropsychiatric Inventory [31], RSS Relationship Satisfaction Scale [32], SF-12 Short Form Health Survey [33], VA LV-VFQ-20 Veterans Affairs Low vision Visual Functioning Questionnaire [34] 
take account of motivation as a potential moderator, or even mediator, of the intervention's impact. Greater independence and communication ability in the $\mathrm{PwD}$ will reduce companions' burden and stress, which will also impact positively the overall wellbeing. Attitudes and knowledge training will support change maintenance and relationship quality [46].

\section{Identification of key uncertainties and developing a framework for the process evaluation}

In Table 2, we identified key uncertainties to address for each SI. We will use a mixed method approach to capture the data for the evaluation. This will involve a variety of instruments, as outlined in Table 3.

\section{Exploration of delivery}

To ascertain whether the SI is delivered (i.e. 'how') and enacted (i.e. 'what') as intended [18], we will examine the fidelity and dose (i.e. duration, number and frequency of SI visits) of the delivered intervention. Due to potential burden on participants, we have chosen not to include an external evaluation of fidelity (i.e. independent observer during sessions). Instead, we will rely on the proxy measure of 'fidelity' as determined by thoroughness of SST training and supervision, use of therapist manual and SST logbook recordings of sessions. We will document the nature of the support offered by the intervention, including the type of corrective devices, the environmental changes to support sensory function, the number and types of referral or signposts to extra-trial services. These data will be captured through participant diaries (the $\mathrm{PwD}$ and their companion) and the SST logbook.

Specifically, the $P w D$ diaries will contain Likert style ratings [50] of acceptability and tolerability of SI visits, including measures of helpfulness, effort, fatigue, understanding and motivation; and how acceptable the corrective sensory devices are. The companions' diaries will capture data relating to how the PwD engages with the visit, how the PwD is adapting to their sensory aids, and how confident the companion feels in supporting the PwD in using the aids. The SST logbooks will contain details of each visit, the components of the SI delivered, participant response to the intervention and skill in managing their aids. Additionally, the SST logbooks will detail how the SI is specifically tailored to the dyad.

We will assess reach through the representativeness of the sites, the recruitment process (refusal rate, attrition rate) and the representativeness of the study population according to the target population [51].

\section{Evaluation of contextual issues}

The SENSE-Cog RCT will take place in several different countries and involve three languages (English, French and Greek). Thus, contextual issues, which are external to the intervention itself, need to be carefully considered. These include differences in language, culture, access to services and the health and social care economy. Context may influence the SST's ability to foster change in the participant dyad's circumstances. For example, for social isolation, the SST may recommend attendance at a local lunch club; however, if transportation is not suitable for individuals with sensory and cognitive impairment, the opportunity to take up the offer will be hampered. Likewise, communication training with companions may be differently received in diverse cultural and linguistic contexts. Thus, the same intervention may have divergent outcomes according to the setting in which it is delivered [18]. The dyadic relationship (between the PwD and their companion) should also be considered because the level of support and quality of relationship may vary among dyads. To capture contextual data, we will collect information from the demographic and outcome measures, the participant dyad diaries, the SST logbook and in-depth qualitative interviews of a sub-sample

Table 2 Identifying key uncertainties to address in the process evaluation for each component of the sensory support intervention

\begin{tabular}{|c|c|}
\hline Sensory support intervention component & Key uncertainty to address \\
\hline Correct visual/auditory impairment & Is vision/hearing impairment actually corrected? \\
\hline Training in correct use of hearing aid/glasses & How well are the devices used by the PwD? \\
\hline Communication training & Is the companion utilising the techniques? \\
\hline Home-based functional assessment & $\begin{array}{l}\text { What are the types and extent of functional } \\
\text { impairment identified by the sensory support therapist? }\end{array}$ \\
\hline Referral to health and social care services & $\begin{array}{l}\text { What types of services are available at each site? } \\
\text { How many referrals were made and actioned? }\end{array}$ \\
\hline $\begin{array}{l}\text { Provision of supplementary sensory devices } \\
\text { in the home environment }\end{array}$ & $\begin{array}{l}\text { How many of these were supplied and how often } \\
\text { and effectively were they used? }\end{array}$ \\
\hline Referral to social/hobby/interest activities & How many referrals were made and actioned? \\
\hline Individualised goal setting & $\begin{array}{l}\text { What is the number and types of goals set with the participant? } \\
\text { How many goals were achieved? } \\
\text { What is the level of apathy present (ascertained only after } \\
\text { final assessment data collected? }\end{array}$ \\
\hline
\end{tabular}


Table 3 Measurement instruments used to collect quantitative and qualitative data to inform the process evaluation

\begin{tabular}{|c|c|c|c|}
\hline \multicolumn{2}{|c|}{ Measurement tool } & \multirow{2}{*}{$\begin{array}{l}\text { Data captured by } \\
\text { whom? About } \\
\text { whom/what? } \\
\text { Researcher about } \\
\text { dyad }\end{array}$} & \multirow{2}{*}{$\begin{array}{l}\text { Timing of } \\
\text { Screening }\end{array}$} \\
\hline 1a) & $\begin{array}{l}\text { Sociodemographic } \\
\text { information }\end{array}$ & & \\
\hline 1b) & Medical history & $\begin{array}{l}\text { Researcher about } \\
\text { dyad }\end{array}$ & Screening \\
\hline 1c) & $\begin{array}{l}\text { HearCheck } \\
\text { PEEK acuity, visual field } \\
\text { confrontation test }\end{array}$ & $\begin{array}{l}\text { Researcher about } \\
\text { PwD }\end{array}$ & Screening \\
\hline 1d) & MoCA & $\begin{array}{l}\text { Researcher about } \\
\text { PwD }\end{array}$ & Screening \\
\hline 1e) & DEMQOL & $\begin{array}{l}\text { Researcher about } \\
\text { PwD }\end{array}$ & Baseline \\
\hline 1f) & BADLs & $\begin{array}{l}\text { Companion about } \\
\text { PwD }\end{array}$ & Baseline \\
\hline 1g) & VA LV-VFQ-20 & $\begin{array}{l}\text { Researcher about } \\
\text { PwD and } \\
\text { companion }\end{array}$ & Baseline \\
\hline $\begin{array}{l}1 \\
\mathrm{~h})\end{array}$ & HHIE-25 & $\begin{array}{l}\text { Researcher about } \\
\text { PwD and } \\
\text { companion }\end{array}$ & Baseline \\
\hline 1i) & $\mathrm{NPI}-12$ & $\begin{array}{l}\text { Researcher and } \\
\text { companion about } \\
\text { PwD }\end{array}$ & Baseline \\
\hline
\end{tabular}

\section{1j) RSS \\ $1 \quad E Q-5 D-5 L$ \\ k) \\ 1I) RUD-Lite}

1 PwD full visual and/or

m) hearing assessment

2) Delivered devices record

3a) PwD diary (acceptability and tolerability of therapy)

3b) PwD diary (acceptance of devices)

3c) Companion diary (acceptability of therapy)
Researcher about Baseline, week 18, week PwD (companion completes on its own)

Researcher about

PwD and

companion

Researcher and companion about PwD

By optometrist and/ Following randomisation or audiologist about PwD

By researcher/
therapist about PwD

By PwD about their own experiences

By PwD about their own experiences

By companion about PwD's
36

Baseline

Baseline, week 18, week 36 Following receipt of glasses and/or hearing aids

Throughout sensory intervention

Throughout sensory intervention

Throughout sensory intervention

Process evaluation (Capability ' $C$ ', Opportunity 'O', Motivation

' $M$ ') and measurement instruments (details)

To assess characteristics of companion and participant (covariates of age, gender, living conditions influencing outcomes)

Dementia sub-type and medication may influence uptake and effectiveness of intervention (eliciting the type of memory impairment and the current medication)

To establish the baseline for ' $\mathrm{C}$ ' and ' $\mathrm{O}$ ' for intervention/optimisation of function (screening for severity of visual and hearing loss of participant)

To ascertain impact of sensory optimisation on cognitive ability as intermediate step (' $C$ ' and ' $M$ ') leading to improved QoL (assessing cognitive level of participant scale (score $\geq 10$ ); also decides whether the participant is appropriate candidate for the study)

The primary outcome of the behavioural change process, resulting from intermediate impacts (assessing quality of life of PwD by addressing all parts of the process evaluation: ' $C$ ', ' $O$ ', 'M')

To ascertain impact of sensory optimisation on functional ability as intermediate step (' $C$ ' and ' $M$ ') leading to improved QoL (assessing functional ability of PwD)

Improvement in sensory input affects ' $\mathrm{C}$ ', ' $\mathrm{O}$ ' and ' $\mathrm{M}$ ', all impacting on intermediate outcomes leading to overall improvement in QoL (assessing for vision- and hearing-related functional ability)

To ascertain impact of sensory optimisation on neuropsychiatric function as intermediate step (' $\mathrm{C}$, ' $\mathrm{O}$ ' and ' $\mathrm{M}$ ') leading to improved $\mathrm{QoL}$ (assessing behavioural and psychological symptoms like anxiety, agitation etc.)

Improved dyadic relationship optimises ' $\mathrm{O}$ ', thus leading to improved QoL (capturing current level of relationship satisfaction between the PwD and study companion, reduce feelings of loneliness)

To measure uptake of health services, enhancing ' $O$ ' (assessing health resource utilisation and capturing the amount and type of support that is in place)

To establish the baseline for ' $\mathrm{C}$ ' and ' $\mathrm{O}$ ' (assessing the degree of the PwD's visual and/or hearing loss)

To ascertain the 'dose' of the intervention (capturing the number and type of corrective sensory devices prescribed by the optometrist and/or audiologist)

To ascertain the utility of the 'correction' component of the intervention from the PwD's perspective (capturing the PwD's ratings on acceptability and tolerability of the SI and acceptability across five indices (helpfulness, fatigue, effort, understanding and motivation) as well as the PwD's view about their corrective sensory devices - also informing ' $C$ ')

To ascertain the utility of the 'correction' component of the intervention from the companion's perspective 
Table 3 Measurement instruments used to collect quantitative and qualitative data to inform the process evaluation (Continued)

\begin{tabular}{lll}
\hline Measurement tool & $\begin{array}{l}\text { Data captured by } \\
\text { whom? About } \\
\text { whom/what? }\end{array}$ & Timing of data captu \\
\hline & experiences & \\
& & \\
3d) Companion diary (adaptation & $\begin{array}{l}\text { By companion } \\
\text { about PwD's } \\
\text { to devices) }\end{array}$ & $\begin{array}{l}\text { Throughout sensory } \\
\text { intervention }\end{array}$ \\
3e) Companion diary & & \\
(companion's confidence) & $\begin{array}{l}\text { By companion } \\
\text { about their own } \\
\text { experiences }\end{array}$ & intervention \\
\end{tabular}

4a) SST logbook (visit record)

4b) SST logbook (response to corrective devices)

4c) SST logbook (delivered components record)

4d) Bangor Goal Setting Inventory

4e) Hearing aid skills and knowledge test and/or glasses/vision skills and knowledge test

4f) GHABP

5) Dyadic experience of $\mathrm{SI}$ (interview)

6) Battery of outcome measures

7a) SST knowledge and skills checklist

7b) SST training log

7c) SST competency checklist

7d) SST supervision log and reflective diary
By therapist about SI Throughout sensory visits

intervention

By therapist about Throughout sensory PWD intervention

By therapist about SI Throughout sensory visits intervention

By therapist about End of sensory PwD intervention

By therapist about Following receipt of PwD

hearing aids/glasses and at end of sensory intervention

By audiologist and At audiology assessment therapist about PwD and at end of sensory intervention

By researcher about Intervention end PWD and companion

By researcher about PwD

Baseline, week 18, week 36

By therapy supervisor about each therapist

By therapy supervisor about each therapist

By therapy supervisor about each therapist

By each therapist and therapy supervisor about themselves
Prior to and post-SST training

Prior to trial commencement

Prior to trial commencement

Throughout trial intervention

Process evaluation (Capability ' $C$ ', Opportunity ' $O$ ', Motivation ' $M$ ') and measurement instruments (details)

(capturing companions' ratings on the PwD's response to the session across five indices of therapy acceptability (interest, autonomy, motivation, emotional responses and mastery) recorded after each session)

To ascertain uptake/adherence with the corrective devices; to assess level of inter-site variability (capturing companions' ratings on the PwD's adaptation to and management and use of the corrective sensory devices)

To capture the companion training component of the intervention; to assess level of inter-site variability (capturing companion's confidence in supporting the PWD's use of their corrective sensory devices)

To ascertain dose of the intervention; to assess level of inter-site variability by:

- Measuring the dose of the SI number of sessions, duration of each session and frequency)

- Quantifying the PwD's capability, opportunity and motivation to use their glasses/hearing aids -Capture adherence to the protocol, i.e. which aspects of the SI were delivered by the SST

To measure little changes within ' $M$ ' (capturing the number and type of goals set by the PwD, and their progress towards these during the $\mathrm{SI}$-address support care needs)

To enhance self-efficacy both in PwD and companion: ' $\mathrm{C}$ ' and ' $\mathrm{O}$ ' (capturing the change in the PwD and companion's skills and knowledge in using their corrective sensory devices through receiving the SI)

To ascertain improved dyadic communication outcomes:

' $C$ ' (capturing self-reported gains from hearing aid use)

To ascertain how the interviews with the dyads were experienced (reflecting upon the positive and negative experiences of intervention for the dyads, perceived benefits/short-comings, suggestions for improvement)

The secondary outcome of the support intervention focusing on behavioural changes (assessing outcomes for participants and companions)

To assess level of inter-site variability as to the SST by:

- A 13-item self-rated questionnaire to identify existing knowledge and skills necessary for the SST role and training needs pre and post-SST training)

- Verifying that the SST has received training in the delivery of the SI components)

- Verify that the SST is competent to undertake the role

- Capturing the number, duration and essence of supervision provided by the senior SST

- Assessing positive and negative aspects of intervention, emerging adaptations, suggestions for improvement 
Table 4 Participant assessment and follow-up visit schedule for the SENSE-Cog Trial

\begin{tabular}{|c|c|c|c|c|c|c|c|c|c|}
\hline & Screening & $\begin{array}{l}\text { Base } \\
\text { line }^{0}\end{array}$ & $\begin{array}{l}\text { Randomi- } \\
\text { sation }\end{array}$ & \multicolumn{3}{|c|}{$\begin{array}{c}\text { Interven- } \\
\text { tion/Care as } \\
\text { usual period }\end{array}$} & $\begin{array}{l}\text { Follow- } \\
\text { up visit }{ }^{0}\end{array}$ & $\begin{array}{c}\text { Fol- } \\
\text { low-up }\end{array}$ & $\begin{array}{l}\text { Final } \\
\text { visit }^{0}\end{array}$ \\
\hline & W-2/W0 & $\begin{array}{l}\text { W2/ } \\
\text { wo }\end{array}$ & wo & $\begin{array}{l}\text { W0- } \\
\text { W7 }\end{array}$ & W8 & $\begin{array}{l}\text { W9- } \\
\text { W18 }\end{array}$ & $\begin{array}{l}\text { W18- } \\
\text { W20 }\end{array}$ & W26 & $\begin{array}{c}\text { W36 } \\
- \\
\text { W38 }\end{array}$ \\
\hline Allowable visit window & & & $\begin{array}{l}\text { max.3 } \\
\text { days } \\
\text { after } \\
\text { baseline }\end{array}$ & & & $\begin{array}{c}+/-2 \\
W\end{array}$ & $+/-2 W$ & $+/-2 W$ & $\begin{array}{c}+/-2 \\
W\end{array}$ \\
\hline Signed informed consent & $\checkmark$ & & & & & & & & \\
\hline Clinical examination $^{1}$ & $\checkmark$ & & & & & & & & \\
\hline $\begin{array}{l}\text { For the PwD (regardless of randomised } \\
\text { allocation): assessments of QoL, func- } \\
\text { tional ability, Global cognitive function- } \\
\text { ing, mental well-being }{ }^{2}\end{array}$ & & $\checkmark$ & & & & & $\checkmark$ & & $\checkmark$ \\
\hline $\begin{array}{l}\text { For the PwD (regardless of randomised } \\
\text { allocation): cost effectiveness assess- } \\
\text { ment }^{2}\end{array}$ & & $\checkmark$ & & & & & $\checkmark$ & & $\checkmark$ \\
\hline $\begin{array}{l}\text { For the companion (regardless of ran- } \\
\text { domised allocation): experience, rela- } \\
\text { tionship with the PwD, depression and } \\
\text { anxiety }^{2}\end{array}$ & & $\checkmark$ & & & & & $\checkmark$ & & $\checkmark$ \\
\hline Intervention group only3: & & & & & & & & & \\
\hline Multi-component Sensory Intervention & & & & & & & & & \\
\hline $\begin{array}{l}\text { Intervention group only: } \\
\text { Qualitative interview }\end{array}$ & & & & & $\checkmark$ & & & & \\
\hline $\begin{array}{l}\text { Care as Usual group only: } \\
\text { Care as Usual }\end{array}$ & & & & & & & & & \\
\hline $\begin{array}{l}\text { Surveillance of clinical AEs } \\
\text { (phone call at W8 and W26) }\end{array}$ & & & & * & $\checkmark$ & * & $\checkmark$ & $\checkmark$ & $\checkmark$ \\
\hline
\end{tabular}

${ }^{0}$ Baseline, follow-up and end visits may be split into two visits, occurring within a maximum of a 2-week window according to the PwD's needs

${ }^{1}$ Clinical examination including assessment of vision impairment (using the PEEK tool), hearing impairment (using the HearCheck device), level of cognitive impairment and other eligibility criteria and medical diagnostic of depression

${ }^{2}$ Baseline (except for the MoCA scale, which will be performed at screening), W18 and W36 (around 2 to $2.5 \mathrm{~h}$ )

${ }^{3}$ The number of sessions may vary from participant to participant but the maximum number of visits will be ten at an average rate of one per week. The order and duration of each component may vary according to the participants' needs, as determined in collaboration with the researcher, the PwD and the companion The remaining weeks until week 18 will allow the SST to revisit and recap previous components, based on the participant's individual needs and goal attainment ${ }^{4}$ In case of dual impairment, the hearing assessment will be done first, followed by the vision assessment, if possible

${ }^{5} \mathrm{~A}$ qualitative interview will be consecutively proposed to each dyad:

- Who experienced completed SI (all intervention visits scheduled by the SST, in addition to the full hearing and vision assessments)

- at the therapist discretion based on their clinical impression of the person be able to provide meaningful feedback

- and who are willing to

- until we reach participation of 60 people (30 interviews), six dyads per site: four dyads with single impairment (two hearing only and two vision only if possible) and two dyads with dual impairment

This qualitative interview will take place within 2 weeks of SI completion. One additional interview, if possible, will be undertaken with a dyad which did not complete the full SI

$W$ week 
of participant dyads ( $n=30$ dyads across the sites), as detailed in the SENSE-Cog trial protocol [16].

\section{Sampling and timing of data collection}

We will collect characteristics of each participant dyad, including gender, age and support structure at the screening visit, and at baseline, week 18 and week 36 (Tables 3 and 4). Following each SI visit (for the active arm), participant diaries and SST logbooks will be completed. The sub-sample qualitative interviews will take place within 2 weeks following the SI (details described in [16]). Training logs for the SSTs were collected prior to study start. SST supervision logs and fidelity checks of the SST logbooks are being collected throughout the trial. SST interviews will be held within 2 weeks after the last intervention visit of the last randomised dyad in each site. Briefly, these interviews will explore the experience of having received the intervention, from the perspective of each member of the dyad. The sample size for this sub-sample was selected to achieve theoretical and data saturation. The interviews from all sites will be analysed by using conventional qualitative content analysis [52] and a grounded theory approach [53].

\section{Analysis}

To avoid biased interpretation, as recommended by the MRC's guidance, we will analyse and explore process data arising from the qualitative interviews and contextual factors before the unblinded trial outcomes are known [18]. We will use the process data to generate specific hypotheses (pre-trial explanation before trial outcomes are revealed) regarding factors that moderate and/or mediate the effect of the SI on outcomes, notably quality of life. This will minimise the risk of 'fishing' for relationships and falsely significant findings due to multiple testing.

Moderator analyses will be undertaken, with appropriate caution, to investigate any influence of the baseline characteristics of the dyads (e.g. age, gender, type of sensory impairment, level of cognitive impairment, type of companion) and country/site effect on the strength and/ or direction of the relationship between the SI and the outcomes. We will undertake mediation analyses to assess the degree to which the impact of the SI on the stated outcomes is a direct effect, or is indirect via the hypothesised mediating factors which will be modelled as latent variables in a structural equation modelling framework.

We will conduct the moderator/mediator analysis only after the final RCT analysis has been completed and the dataset has been un-blinded. We will do this regardless of whether the SI has a significant direct impact on the primary outcome.
We will apply a regression framework, using newer methods and statistical models $[54,55]$ that improve on traditional approaches (e.g. [56]). These models can become complex, particularly when controlling for multiple covariates. Thus, depending upon the number and complexity of the hypotheses to be tested, we will assess whether it will be better to analyse each factor separately, or to combine sets of moderators and/or mediators into a 'conditional process analysis' [54]. We will conduct separate analyses for those variables available in both trial arms, and those available in the intervention group only (e.g. related to the SI). The latter analyses will help to identify process measures that are part of the SI and may moderate its efficacy (for e.g., number of SST visits, SST experience and fidelity), using appropriate techniques [57].

\section{Discussion}

The process evaluation of the SENSE-Cog RCT will appraise several important aspects of the delivery of the intervention, the context of delivery and the hypothesised causative mechanisms. These issues are key to interpreting the effectiveness outcomes of the trial and, if outcomes are positive, to assist in understanding implications for scale-up in clinical settings. The SI will have multiple interacting components: assessing and correcting hearing and vision impairment (hearing aids and/or glasses lenses), training in the use of the devices, enhancing communication within the dyad, optimising the home sensory environment and supporting engagement in health and wellbeing opportunities in the community, including social integration and external support services.

A key challenge in delivering the SENSE-Cog SI is to maintain standardisation and rigour when implementing such a complex intervention across five different European sites. The SENSE-Cog SI is ambitious in its vision in addressing three co-morbidities simultaneously-cognitive impairment, hearing loss and vision loss-and to assess the impact of a psychosocial intervention on managing these impairments. We will aim to capture the cultural, social and economic nuances of the respective European study sites whilst deriving results that can be applied in a pan-European context. To ensure that we capture the cultural differences from the qualitative interviews, we will keep translation to a minimum, as recommended by Haak et al. [58].

The theoretical model with causal relationships will be informed by the mediation analyses. The elicited data will also enable us to 'test' the theoretical model of the COM-B by addressing the key uncertainties listed in Table 1 . This will be the first opportunity to evaluate empirically the COM-B model in a RCT setting, as demonstrated in Fig. 1. 


\section{Strengths and limitations of the study}

A strength of our study protocol includes the use of a mixed method approach, including both qualitative and quantitative measures, to carefully explore the "how and why' of the intervention. Another strength of our approach includes the sound theoretical framework on which the intervention was developed, and the iterative manner in which it was modified and field-tested [12, 15] before arriving at the final version of the intervention, ready for full scale effectiveness testing. A limitation (although also a potential strength) is the significant degree of variability in the study sites due to the different EU contexts in which the programme takes place, as well as the variability of the intervention offered to each participant dyad, resulting from the tailored approach.

\section{Reporting and dissemination}

We will report the results of the process evaluation described here using a combination of reporting guidance, including CONSORT [59] and COREQ (for the qualitative outcomes) [60] as well as statistical methods for mediators and moderators [61]. We will submit the findings to an open-access journal, as per the requirements of the funder. As recommended by others [51], we have described our protocol in advance to foster transparency in reporting and to help the development and evaluation of complex psychosocial interventions for PwD, an emerging area of health services research. Finally, we will link our outputs related to the SENSE-Cog trial through the SENSE-Cog programme website (www. sense-cog.eu).

\section{Ethics approval and consent to participate}

In Manchester, the study received final approval (version 3.0) by the NW Haydock ethics committee on 22 January 2018 and obtained sponsor approval on 8 March 2019. In Nicosia, the study received favourable opinion on 27 September 2016 from the Cyprus National Bioethics Committee. In Athens, the Local Ethics Committee of Health Sciences and Scientific Committee of the Eginition Hospital of the National and Kapodistrian University of Athens ethics committee granted a favourable opinion on 24 January 2018. In Dublin, the Saint James Hospital/AMNCH Research Ethics Committee gave approval on the 25 October 2018. In Nice, the "Comité de Protection des personnes Sud Est I" gave a favourable opinion on 12 July 2018. Written consent is collected from the participants eligible for the study, using procedures in accordance with the national guidance regarding informed consent and clinical research (for individuals with or without capacity to consent) in each of the participating countries (detailed in Regan et al. [16]). All researchers have been fully trained in Good Clinical Practice (GCP) and mental capacity assessment skills and follow national guidance in their respective countries, such as the Mental Capacity Act (2005) in the UK. If a person lacks capacity, a nominated consultee will be asked to deem whether it is in the PwD's best interests to participate.

\section{Trial status}

This process evaluation is based on the SENSE-Cog RCT protocol version 4.0 of 16 November 2018. The overall SENSE-Cog research programme started in January 2016 and the SENSE-Cog RCT (Work Package 3.2) started recruitment in summer 2018. Recruitment is expected to end in December 2020. The first qualitative interviews with participants took place in November 2018. The process evaluation will start in following the last W36 assessment of the first randomized participant dyad.

\section{Supplementary information}

Supplementary information accompanies this paper at https://doi.org/10. 1186/s13063-020-4135-4

Additional file 1. Good Reporting of A Mixed Methods Study (GRAMMS).

\section{Abbreviations}

AE: Adverse events; COM-B: Capability, opportunity, motivation-behavioural change wheel; MRC: Medical research council; PwD: People with dementia; RCT: Randomized controlled trial; SI: Sensory intervention; SST: Sensory support therapist

\footnotetext{
Acknowledgements

The authors would like to acknowledge and thank the SENSE-Cog Research User Group, which consists of stakeholders with dementia and sensory impairment and their companions and is coordinated by Jahanara Miah. Their input into all stages of development of the intervention, the protocol and the study materials has been invaluable. Lynne McCrae, the University of Manchester's Research Practice Governance Coordinator, was very helpful in providing feedback on the protocol. We also acknowledge and thank Francine Jury and Jemma Regan for support in coordination.

SENSE-Cog WP3.2: Harvey Abrams, Nathalie Chaghil-Boissière, Anna Pavlina

Charalambous, Fidéline Colllin, Fofi Constantinidou, Lisa Crosby, Piers Dawes, Eric Frison, Camille Gilbert, Mark Hann, Emma Hooper, Ines Himmelsbach, Evangelia Kontogianni, Brian Lawlor, Iracema Leroi, Sarah Marie, Susana Montecelo, Antonios Politis, Otilia Postea, David Reeves, David Renaud, Zoe Simkin, Monique Termote, Chryssoula Thodi, Lucas Wolski.

${ }^{7}$ Starkey Hearing Technologies

${ }^{8}$ Bordeaux Population health Research Centre

${ }^{9}$ European University of Cyprus

${ }^{10}$ The University of Manchester, Manchester Centre for Health Psychology,

${ }^{11}$ Centre Hospitalier Universitaire de Bordeaux

${ }^{12}$ Université de Bordeaux

${ }^{13}$ National and Kapodistrian University of Athens

${ }^{14}$ St James' Hospital, Dublin

${ }^{15}$ Essilor International

${ }^{16}$ ARTTIC Consultancy and Management

${ }^{17}$ The University of Manchester, Division of Population Health, Health

Services Research \& Primary Care

${ }^{18}$ French Institute of Health and Medical Research
}

Authors' contributions

$I L, E F, F C, M H, D R, E H$ and $Z S$ and $L W$ were responsible for the overall development of an ethically sound protocol. IL, EF, MH, LW, EH, ZS and CA 
led the design of the process evaluation. All authors contributed to the drafting, critical revision and final approval of the manuscript.

\section{Funding}

The SENSE-Cog project has received funding from the European Union's Horizon 2020 research and innovation programme under grant agreement 668648. This process evaluation protocol comprises part of the work undertaken in Work Package 3 of the overall SENSE-Cog programme. The design of the study and collection, analysis and interpretation of data and in writing the manuscript were all undertaken independently of the funding body. The funding body had no influence on any aspect of the study.

\section{Availability of data and materials}

Data generated or analysed during this study will be included in the article reporting the results that will be shared through scientific articles and international conferences.

\section{Competing interests}

The authors declare that they have no competing interests.

\section{Author details}

${ }^{1}$ Global Brain Health Institute, School of Medicine, Trinity College Dublin, Trinity College Institute of Neurosciences, Room 0.60, Lloyd Building, Dublin 2, Ireland. 'Division of Neuroscience and Experimental Psychology, University of Manchester and the Manchester Academic Health Sciences Centre, Manchester, UK. ${ }^{3}$ Manchester Centre for Health Psychology, School of Psychological Sciences, University of Manchester, Manchester, UK. ${ }^{4}$ Bordeaux Population Health Center, Univ. Bordeaux, INSERM, EUCLID/F-CRIN Clinical Trials Platform, CHU Bordeaux, F-33000 Bordeaux, France. ${ }^{5}$ Division of Population Health, Health Services Research \& Primary Care, University of Manchester, Manchester, UK. ${ }^{6}$ Institute of Applied Research, Development and Continuing Education, Catholic University of Applied Sciences, Freiburg, Germany.

Received: 23 July 2019 Accepted: 3 February 2020

Published online: 24 February 2020

\section{References}

1. Fortunato S, Forli F, Guglielmi V, De Corso E, Paludetti G, Berrettini S, et al. A review of new insights on the association between hearing loss and cognitive decline in ageing. Acta Otorhinolaryngo. 2016:36(3):155-66.

2. Rutherford BR, Brewster K, Golub JS, Kim AH, Roose SP. Sensation and psychiatry: Linking age-related hearing loss to late-life depression and cognitive decline. Am J Psychiatry. 2018;175(3):215-24.

3. Lawrence V, Murray J, Banerjee S, Ffytche D. The experiences and needs of people with dementia and serious visual impairment: a qualitative study. London: Thomas Pocklington Trust; 2008.

4. Maharani A, Pendleton N, Leroi I. Hearing impairment, loneliness, social isolation, and cognitive function: Longitudinal analysis using English Longitudinal Study on Ageing. Am J Geriat Psychiat. 2019;27(12):1348-56.

5. McKeefry D, Bartlett R. Improving vision and eye health care to people with dementia. London: Thomas Pocklington Trust; 2010.

6. Elliott AF, McGwin G, Owsley C. Health-related quality of life and visual and cognitive impairment among nursing-home residents. Brit J Ophthalmol. 2009:93(2):240-3.

7. Lupsakko T, Mantyjarvi M, Kautiainen H, Sulkava R. Combined hearing and visual impairment and depression in a population aged 75 years and older. Int J Geriatr Psych. 2002;17(9):808-13.

8. McDonnall MC. The effects of developing a dual sensory loss on depression in older adults: a longitudinal study. J Aging Health. 2009;21(8):1179-99.

9. Dawes P, Wolski L, Himmelsbach I, Regan J, Leroi I. Interventions for hearing and vision impairment to improve outcomes for people with dementia: a scoping review. Int Psychogeriatr. 2019;31(2):203-21.

10. Leroi I, Wolski L, Charalambous AP, Constantinidou F, David R, Dawes P, Hann M, Himmelsbach I, Miah J, Payne M, Simkin Z, Thodi C, Yeung W, Yohannes AM. Support care needs of people with dementia and hearing and vision impairment: A European perspective. In: ACRM 96th Annual Conference, Progress in Rehabilitation Research. Chicago: American Congress of Rehabilitation Medicine; 2019.

11. Leroi I, Himmelsbach I, Wolski L, Littlejohn J, Jury F, Parker A, et al. Assessing and managing concurrent hearing, vision and cognitive impairments in older people: an international perspective from healthcare professionals. Age Ageing. 2019;48(4):580-7.

12. Leroi I, Pye A, Armitage CJ, Charalambous AP, Constantinidou F, Helmer C, et al. Research protocol for a complex intervention to support hearing and vision function to improve the lives of people with dementia. Pilot Feasibility Stud. 2017:3:38.

13. Regan J, Dawes P, Pye A, Armitage CJ, Hann M, Himmelsbach I, et al. Improving hearing and vision in dementia: protocol for a field trial of a new intervention. BMJ Open. 2017;7(11):1-12.

14. Hooper E, Simkin Z, Abrams H, Camacho E, Charalambous AP, Collin F, et al. Feasibility of an intervention to support hearing and vision in dementia: The SENSE-Cog field trial. J Am Geriatr Soc. 2019;67(7):1472-7.

15. Leroi I, Simkin Z, Hooper E, Wolski L, Abrams H, Armitage CJ, et al. Impact of an intervention to support hearing and vision in dementia: The SENSE-Cog field trial. Int J Geriatr Psych. 2019:1-10.

16. Regan J, Frison E, Collin F, Dawes P, Hann M, Himmelsbach I, et al. Individualised sensory intervention to improve quality of life in people with dementia and their companions (SENSE-Cog trial): study protocol for a randomised controlled trial. Trials. 2019:20:1-15.

17. Smith SC, Lamping DL, Banerjee S, Harwood R, Foley B, Smith P, et al. Measurement of health-related quality of life for people with dementia: development of a new instrument (DEMQOL) and an evaluation of current methodology. Health Technol Assess. 2005;9(10):1-93 iii-iv.

18. Moore GF, Audrey S, Barker M, Bond L, Bonell C, Hardeman W, et al. Process evaluation of complex interventions: Medical Research Council guidance. BMJ. 2015:350:1-7.

19. Craig P, Dieppe P, Macintyre S, Michie S, Nazareth I, Petticrew M. Developing and evaluating complex interventions: the new Medical Research Council guidance. BMJ. 2008;337(7676):1-6

20. Barratt H, Campbell M, Moore L, Zwarenstein M, Bower P. Randomised controlled trials of complex interventions and large-scale transformation of service. In: Raine R, Fitzpatrick R, Barratt H, Bevan G, Black N, Boaden R, et al, editors. Health Services and Delivery Research; 2016. p. 19-31.

21. Howarth E, Devers K, Moore G, O'Cathain A, Dixon-Woods M. Contextual issue and qualitative research. In: Raine R, Fitzpatrick R, Barratt $H$, Bevan G, Black N, Boaden R, et al., editors. Health Services and Delivery Research; 2016. p. 205-120.

22. Bucks RS, Ashworth DL, Wilcock GK, Siegfried K. Assessment of activities of daily living in dementia: Development of the Bristol activities of daily living scale. Age Ageing. 1996;25(2):113-20.

23. Clare L, Hindle JV, Jones IR, Thom JM, Nelis SM, Hounsome B, et al. The AgeWell study of behavior change to promote health and wellbeing in later life: study protocol for a randomized controlled trial. Trials. 2012;13:1-9.

24. Smith SC, Lamping DL, Banerjee S, Harwood RH, Foley B, Smith P, Cook JC, Murray J, Prince M, Levin E, Mann A, Knapp M. Development of a new measure of health-related quality of life for people with dementia: DEMQOL. Psychol Med. 2006:37:737-46.

25. Schofield HL, Murphy B, Herrman HE, Bloch S, Singh B. Family caregiving: Measurement of emotional well-being and Various aspects of the caregiving role. Psychol Med. 1997;27(3):647-57.

26. Zigmond AS, Snaith RP. The hospital anxiety and depression scale. Acta Psychiat Scand. 1983;67(6):361-70.

27. Saunders GH, Morse-Fortier C, McDermott DJ, Vachhani JJ, Grush LD, Griest $S$, et al. Description, normative data, and utility of the hearing aid skills and knowledge test. J Am Acad Audiol. 2018;29(3):233-42.

28. Newman CW, Weinstein BE. Judgements of perceived hearing handicap by hearing-impaired elderly men and their spouses. J Acad Rehabilitative Audiol. 1986;19:109-15.

29. Goldberg DP, Blackwell B. Psychiatric illness in general practice - a detailed study using a new method of case identification. BMJ. 1970;2(5707):439.

30. Nasreddine ZS, Phillips NA, Bedirian V, Charbonneau S, Whitehead V, Collin I, et al. The Montreal Cognitive Assessment, MoCA: A brief screening tool for mild cognitive impairment. J Am Geriatr Soc. 2005;53(4):695-9.

31. Cummings JL. The Neuropsychiatric Inventory: assessing psychopathology in dementia patients. Neurology. 1997:48(5 Suppl 6):S10-6.

32. Burns D. Relationship Satisfaction Scale 1983. Ten days to self-esteem. New York: Harper Collins; 1993.

33. Brazier JE, Roberts J. The estimation of a preference-based measure of health from the SF-12. Med Care. 2004;42(9):851-9.

34. Stelmack JA, Massof RW Using the VA LV VFO-48 and LV VFO-20 in low vision rehabilitation. Optom Vis Sci. 2007;84(8):705-9. 
35. Mulhern B, Rowen D, Brazier J, Smith S, Romeo R, Tait R, et al. Development of DEMQOL-U and DEMQOL-PROXY-U: generation of preference-based indices from DEMQOL and DEMQOL-PROXY for use in economic evaluation. Health Technol Asses. 2013;17(5):1-160.

36. Michie S, van Stralen MM, West R. The behaviour change wheel: a new method for characterising and designing behaviour change interventions. Implement Sci. 2011;6:42.

37. Wong LLN, Yu JKY, Chan SS, Tong MCF. Screening of cognitive function and hearing impairment in older adults: A preliminary study. Biomed Res Int. 2014:2014:1-7.

38. Maharani A, Dawes P, Nazroo J, Tampubolon G, Pendleton N, Grp S-CW. Cataract surgery and age-related cognitive decline: A 13-year follow-up of the English Longitudinal Study of Ageing. Plos One. 2018;13(11):e0204833.

39. Ghiringhelli R, lorio MCM. Hearing aids and recovery times: a study according to cognitive status. Braz J Otorhinolar. 2013;79(2):177-84.

40. Martini A, Castiglione A, Bovo R, Vallesi A, Gabelli C. Aging, cognitive load, dementia and hearing loss. Audiol Neurootol. 2014;19(Suppl 1):2-5.

41. Dawes P, Emsley R, Cruickshanks KJ, Moore DR, Fortnum H, EdmondsonJones $\mathrm{M}$, et al. Hearing loss and cognition: the role of hearing AIDS, social isolation and depression. PLoS One. 2015;10(3):e0119616.

42. Nieman CL, Marrone N, Mamo SK, Betz J, Choi JS, Contrera KJ, et al. The Baltimore HEARS Pilot Study: An affordable, accessible, CommunityDelivered Hearing Care Intervention. Gerontologist. 2017;57(6):1173-86.

43. Acton JH, Molik B, Court H, Margrain TH. Effect of a home visit-based low vision rehabilitation intervention on visual function outcomes: An exploratory randomized controlled trial. Invest Ophth Vis Sci. 2016;57(15): 6662-7.

44. Kreeger JL, Raulin ML, Grace J, Priest BL. Effect of hearing enhancement on mental status ratings in geriatric psychiatric-patients. Am J Psychiat. 1995; 152(4):629-31.

45. Rogers MA, Langa KM. Untreated poor vision: a contributing factor to latelife dementia. Am J Epidemiol. 2010;171(6):728-35.

46. Ogden J, Hills L. Understanding sustained behavior change: the role of life crises and the process of reinvention. Health (London). 2008;12(4):419-37.

47. EuroQol G. EuroQol--a new facility for the measurement of health-related quality of life. Health Policy. 1990;16(3):199-208.

48. Wimo A, Jonsson L, Zbrozek A. The resource utilization in dementia (RUD) instrument is valid for assessing informal care time in community-living patients with dementia. J Nutr Health Aging. 2010;14(8):685-90.

49. Gatehouse S. Glasgow Hearing Aid Benefit Profile : Derivation and Validation of a Client-centered Outcome Measure for Hearing Aid Services. J Am Acad Audiol. 1999;10:80-103.

50. Likert R. A technique for the measurement of attitudes. Arch Psychol. 1932; 22(140):5-55.

51. Liu H, Lindley R, Alim M, Felix C, Gandhi DB, Verma SJ, et al. Protocol for process evaluation of a randomised controlled trial of family-led rehabilitation post stroke (ATTEND) in India. BMJ Open. 2016;6(9):e012027.

52. Hsieh HF, Shannon SE. Three approaches to qualitative content analysis. Qual Health Res. 2005;15(9):1277-88.

53. Charmaz K. In: Silverman D, editor. Constructing grounded theory: A practical guide through qualitative analysis. London: SAGE Publications Ltd; 2006.

54. Hayes AF, Rockwood NJ. Regression-based statistical mediation and moderation analysis in clinical research: Observations, recommendations, and implementation. Behav Res Ther. 2017;98:39-57.

55. Fairchild AJ, MacKinnon DP. A general model for testing mediation and moderation effects. Prev Sci. 2009;10(2):87-99.

56. Baron RM, Kenny DA. The moderator mediator variable distinction in social psychological-research - conceptual, strategic, and statistical considerations. J Pers Soc Psychol. 1986;51(6):1173-82.

57. Dunn G, Emsley R, Liu H, Landau S, Green J, White I, et al. Evaluation and validation of social and psychological markers in randomised trials of complex interventions in mental health: a methodological research programme. Health Technol Assess. 2015;19(93):1-115 v-vi.

58. Haak M, Himmelsbach I, Granbom M, Lofqvist C. Cross-national and multilanguage qualitative research: challenges and recommendations. Brit J Occup Ther. 2013;76(7):333-6.

59. Moher D, Hopewell S, Schulz KF, Montori V, Gotzsche PC, Devereaux PJ, et al. CONSORT 2010 explanation and elaboration: updated guidelines for reporting parallel group randomised trials. Int J Surg. 2012;10(1):28-55.
60. Tong A, Sainsbury P, Craig J. Consolidated criteria for reporting qualitative research (COREQ): a 32-item checklist for interviews and focus groups. Int J Qual Health Care. 2007;19(6):349-57.

61. Lang TA, Secic M. How to report statistics in medicine: Annotated guidelines for authors, editors, and reviewers. 2nd ed. Philadelphia: American College of Physicians; 2006.

\section{Publisher's Note}

Springer Nature remains neutral with regard to jurisdictional claims in published maps and institutional affiliations.
Ready to submit your research? Choose BMC and benefit from:

- fast, convenient online submission

- thorough peer review by experienced researchers in your field

- rapid publication on acceptance

- support for research data, including large and complex data types

- gold Open Access which fosters wider collaboration and increased citations

- maximum visibility for your research: over $100 \mathrm{M}$ website views per year

At BMC, research is always in progress.

Learn more biomedcentral.com/submissions 